\title{
Further evidence for the presence of a neutron star in 4U 2206+54. INTEGRAL and VLA observations *
}

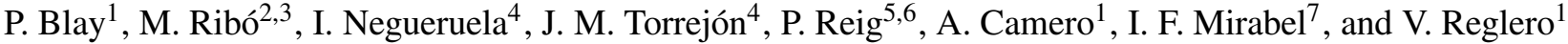 \\ 1 Institut de Ciència dels Materials, Universitat de València, PO Box 22085, 46071 Valencia, Spain \\ e-mail: [pere.blay; ascension.camero; victor.reglero]@uv.es \\ 2 DSM/DAPNIA/Service d'Astrophysique, CEA/Saclay, Bât. 709, L'Orme des Merisiers, 91191 Gif-sur-Yvette Cedex, France \\ 3 AIM - Unité Mixte de Recherche CEA - CNRS - Université Paris VII - UMR 7158, France \\ e-mail: mribo@discovery.saclay.cea.fr \\ ${ }^{4}$ Departamento de Física, Ingeniería de Sistemas y Teoría de la Señal, Escuela Politécnica Superior, Universitat d'Alacant, \\ Ap. 99, 03080 Alicante, Spain \\ e-mail: [ignacio; jmt] @dfists.ua.es \\ 5 IESL, Foundation for Research and Technology, 71110 Heraklion, Crete, Greece \\ ${ }^{6}$ University of Crete, Physics Department, PO Box 2208, 71003 Heraklion, Crete, Greece \\ e-mail: pau@physics.uoc.gr \\ 7 European Southern Observatory, Alonso de Córdova 3107, Vitacura, Casilla 19001, Santiago 19, Chile \\ e-mail: fmirabel@eso.org
}

Received 19 October 2004 / Accepted 29 March 2005

\begin{abstract}
The majority of High Mass X-ray Binaries (HMXBs) behave like X-ray pulsars, revealing that they contain a magnetised neutron star. Among the four HMXBs showing neither pulsations nor the characteristics of accreting black holes, there is the unusual HMXB 4U 2206+54. Here we present contemporaneous high-energy and radio observations of this system conducted with INTEGRAL and the VLA, in order to unveil its nature. The high-energy spectra show clear indications of the presence of an absorption feature at $\sim 32 \mathrm{keV}$. This is the third high-energy observatory to reveal marginal evidence of this feature, giving strong support to the existence of a cyclotron resonance scattering feature, which implies a magnetic field of $3.6 \times 10^{12} \mathrm{G}$. On the other hand, the source is not detected at centimetre radio wavelengths with a $3 \sigma$ upper limit of $0.039 \mathrm{mJy}$. The expected radio emission for an accreting black hole in the low/hard state, inferred from X-ray flux measurements, would be at least 60 times greater than the measured upper limit. Both results firmly indicate that, in spite of the absence of pulsations, $4 \mathrm{U} 2206+54$ hosts a magnetic accreting neutron star, the first one not to be observed as an X-ray pulsar.
\end{abstract}

Key words. stars: individual: 4U 2206+54 - X-rays: binaries - radio continuum: stars - accretion, accretion disks magnetic fields - stars: binaries: close

\section{Introduction}

The vast majority of High Mass X-ray Binaries (HMXBs) harbour X-ray pulsars (cf. Bildsten et al. 1997) believed to be young neutron stars with relatively strong magnetic fields $\left(B \sim 10^{12} \mathrm{G}\right)$. An important fraction of them are wind-fed systems, in which the pulsar accretes from the radiative wind of an OB supergiant (perhaps, in some cases, a Wolf-Rayet star).

Among the handful of HMXBs not displaying X-ray pulsations, only three show the typical characteristics of accreting black holes (LMC X-1, LMC X-3, and Cyg X-1). In four other HMXBs, pulsations have not been discovered in

* Based on observations with INTEGRAL, an ESA project with instruments and science data centre funded by ESA member states (especially the PI countries: Denmark, France, Germany, Italy, Switzerland, Spain), Czech Republic, and Poland, and with the participation of Russia and the USA. spite of intensive searches, but there is no strong evidence identifying the accreting object as a black hole. There is no a priori reason to attribute the lack of pulsations in all these systems to any particular characteristic, and different models have indeed been proposed to explain some of them. There have been suggestions, for instance, that $2 \mathrm{E} 0236.6+6101$, whose counterpart is the B0 Ve star LS I $+61^{\circ} 303$, and RX J1826.2-1450, identified with the O6.5 V((f)) star LS 5039, may not be accreting binaries after all, but instead X-ray sources powered by rotational energy from a young non-accreting neutron star (Maraschi \& Treves 1981; Martocchia et al. 2005); although the presence of relativistic radio jets points towards the accretion scenario (Massi et al. 2004; Paredes et al. 2000). In the case of $4 \mathrm{U} 1700-37$, optically identified with the O6.5 Iaf+ star HD 153919, a compact object of unknown nature and mass $M_{\mathrm{X}}=2.4 \pm 0.3 M_{\odot}$ accretes material from the wind of the massive supergiant (Clark et al. 2002). 
The fourth HMXB not displaying pulsations is $4 \mathrm{U} 2206+54$, identified with the O9p star BD $+53^{\circ} 2790$ (Negueruela \& Reig 2001; henceforth NR01). The relatively high X-ray luminosity of $4 \mathrm{U} 2206+54, L_{\mathrm{X}} \sim 10^{35} \mathrm{erg} \mathrm{s}^{-1}$ (at an estimated distance of $3 \mathrm{kpc}$; NR01), combined with its spectral shape, makes the presence of a neutron star or a black hole in the system almost unavoidable. There are reasons to believe that the compact object in this system is a neutron star (NR01; Torrejón et al. 2004; Masetti et al. 2004), but the possibility of a black-hole has not been ruled out completely by previous observations. Analysis of the RXTE/ASM X-ray lightcurve revealed a $9.6 \mathrm{~d}$ periodicity, which is likely to be the orbital period of a compact object (Corbet \& Peele 2001; Ribó et al. 2005). Moreover, the X-ray lightcurve displays short aperiodic variability, with changes in the flux by a factor $\sim 10$ over timescales of minutes (Saraswat \& Apparao 1992; NR01), which are typically seen in wind-fed systems, presumably as a consequence of stochastic variability in the wind.

Interestingly, many high-energy sources not showing pulsations are microquasars (containing either black holes or neutron stars), while pulsating sources do not show significant radioemission (Fender \& Hendry 2000). 4U 2206+54 shares many characteristics with the well-known microquasar LS 5039 (Paredes et al. 2000, 2002). Both systems contain a nonsupergiant late O-type star (Clark et al. 2001) and a compact object that does not show pulsations (Ribó et al. 1999; Reig et al. 2003) orbiting in a relatively close orbit when compared to the majority of HMXBs, and both systems show evidence of wind-fed accretion (NR01; McSwain et al. 2004) with X-ray luminosities in the range $10^{34}-10^{35} \mathrm{erg} \mathrm{s}^{-1}$. However, an inspection of the VLA Sky Survey (NVSS, Condon et al. 1998) reveals no radio emission up to a $3 \sigma$ upper limit of $1 \mathrm{mJy}$ from $4 \mathrm{U} 2206+54$. The apparent lack of both radio emission and pulsations does not fit within either the typical scenario of a pulsar in an HMXB or the microquasar scenario, so a deeper multiwavelength approach is necessary.

In this work we present new INTEGRAL and VLA observations of the source during the periods December 2002September 2004 and May-June 2003, respectively. The possible presence of a cyclotron line, already suggested by the data from RXTE and BeppoSAX (Torrejón et al. 2004; Masetti et al. 2004), and the absence of radio emission are discussed.

\section{Observations and data analysis}

\subsection{High-energy observations}

INTEGRAL is a joint European mission in flight from October 2002, with three on-board high-energy instruments: the Imager on Board INTEGRAL Spacecraft (IBIS), coupled with the INTEGRAL Soft Gamma-Ray Imager (ISGRI) and the Pixellated Imaging Caesium Iodide Telescope (PICsIT), sensitive to $\gamma$-rays from $15 \mathrm{keV}$ up to $10 \mathrm{MeV}$; the SPectrometer in INTEGRAL (SPI), optimised for spectroscopy in the $20 \mathrm{keV}-8 \mathrm{MeV}$ energy range; and the
Joint European Monitor-X (JEM-X) with twin X-ray monitors, which provides information at lower energies (3-35 keV). An Optical Monitoring Camera (OMC) gives source fluxes in the $V(550 \mathrm{~nm})$ band and complements the 3 high-energy instruments. All 4 instruments are co-aligned, allowing simultaneous observations in a very wide energy range. A detailed description of the mission can be found in Winkler et al. (2003).

INTEGRAL observed the region around 4U 2206+54 on several occasions during its first 22 months of Galactic Plan Survey scans (GPSs), i.e., from December 2002 to September 2004. We present in Table 1 a summary of all the INTEGRAL revolutions during which the source was inside the Field Of View (FOV) of ISGRI. In total, the source was observed by ISGRI for $\sim 337 \mathrm{ks}$, but it was significantly detected only for $27 \mathrm{ks}^{1}$. For revolutions 70, 74, 145, and 189 only an upper limit is given because the source had quite a marginal position in the FOV of ISGRI so the detection is not significant enough. The JEM-X FOV is smaller and thus data were only collected during those revolutions when INTEGRAL pointed close enough to the source (marked in Table 1 with the $\dagger$ symbol) $)^{2}$. Only a few pointings in May and June 2003 fulfill this requirement. Although SPI has the largest FOV, it cannot acquire enough information with one exposure due to the detector design. To achieve a $S / N \sim 10$ for a source like $4 \mathrm{U} 2206+54$, SPI would need around $300 \mathrm{ks}$. Nevertheless, using SPIROS in TIMING mode (see Skinner \& Connell 2003) a light curve was attained in the $20-40 \mathrm{keV}$ energy range. The obtained flux values are in good agreement with the ISGRI data, but have larger uncertainties in comparison. Therefore, no data from SPI were used in this analysis. Data reduction were performed with the standard Offline Analysis Software (OSA) version 4.0, available from the INTEGRAL Science Data Centre (ISDC) ${ }^{3}$. A detalied description of the software can be found in Goldwurm et al. (2003), Diehl et al. (2003), Westergaard et al. (2003) and references therein.

Archived RXTE/PCA lightcurves of four long observations made between October 12 and 20, 2001, have also been used. The Proportional Counter Array, PCA, consists of five coaligned Xenon proportional counter units with a total effective area of $\sim 6000 \mathrm{~cm}^{2}$ and nominal energy range from $2 \mathrm{keV}$ to over $60 \mathrm{keV}$ (Jahoda et al. 1996). In order to produce lightcurves only the top Xenon layer in standard2 mode was used. The durations of these observations range from 17.7 to $29.8 \mathrm{ks}$ and the complete integration time spans $\sim 100 \mathrm{ks}$. A more detailed description of these observations is given in Torrejón et al. (2004).

\footnotetext{
1 A detection is considered for ISGRI when the detection level, which is given by the software package and is not directly related to the number of $\sigma$ above background, lies above a value of 8. For the typical exposure times of GPS pointings $(\sim 2 \mathrm{ks})$, the sensitivity limit of ISGRI lies around 2.5 count $\mathrm{s}^{-1}$ at $20-40 \mathrm{keV}$ energy range.

2 A detection is considered for JEM-X when the detection level, which is given by the software package and is not directly related to the number of $\sigma$ above background, lies above a value 20 .

${ }^{3}$ http://isdc.unige.ch/index.cgi?Soft+download
} 
Table 1. Summary of ISGRI observations of the field around $4 \mathrm{U} 2206+54$ during the INTEGRAL GPS. We list the revolution number, the corresponding dates, the intervals of time when the source was in the FOV of ISGRI during these revolutions in Modified Julian Days, the total on-source time within these intervals, the amount of time in which the source was detected, the mean count rate and its formal error obtained in the $20-40 \mathrm{keV}$ energy range, and the detection level value given by the software package (where a significant detection is found for a detection level equal or above a value of 8). For those revolutions where the significance of the detection was between $1 \sigma$ and $3 \sigma$ (namely, revolutions 70 , 74,145 , and 189), we give $3 \sigma$ upper limits by scaling the detected count rates, assuming that a $3 \sigma$ detection would correspond to a detection level of 8 . The $\uparrow$ symbol indicates those revolutions in which data from JEM-X allowed detection of the source.

\begin{tabular}{|c|c|c|c|c|c|c|}
\hline Rev. & Date & MJD & $\begin{array}{c}\text { On-source time } \\
(\mathrm{ks})\end{array}$ & $\begin{array}{c}\text { Detected time } \\
(\mathrm{ks})\end{array}$ & $\begin{array}{c}\text { Mean count rate } \\
\left(\text { count s}^{-1}\right)\end{array}$ & Detection level \\
\hline 26 & 2002 Dec. $30-2003$ Jan. 1 & $52638.43-52640.07$ & 14 & - & - & - \\
\hline 31 & 2003 Jan. $14-16$ & $52653.32-52655.90$ & 12 & - & - & - \\
\hline 47 & 2003 Mar. 03-05 & $52701.15-52701.27$ & 8 & - & - & - \\
\hline 51 & 2003 Mar. $15-17$ & $52714.85-52714.96$ & 8 & 2.2 & $4.1 \pm 0.4$ & 9.0 \\
\hline 54 & 2003 Mar. 24-26 & $52722.85-52722.93$ & 6 & 2.3 & $4.1 \pm 0.4$ & 8.0 \\
\hline 55 & 2003 Mar. 27-29 & $52727.64-52727.67$ & 4 & - & - & - \\
\hline 59 & 2003 Apr. 08-10 & $52737.03-52737.10$ & 4 & - & - & - \\
\hline 62 & 2003 Apr. 17-19 & $52746.01-52746.04$ & 10 & 7.3 & $3.9 \pm 0.4$ & 8.6 \\
\hline $67 \dagger$ & 2003 May 01-04 & $52761.26-52762.45$ & 8 & 6.5 & $5.9 \pm 0.5$ & 12.6 \\
\hline 70 & 2003 May $10-13$ & $52769.93-52770.10$ & 12 & - & $<7.1$ & 4.1 \\
\hline 74 & 2003 May 22-25 & $52781.92-52782.12$ & 14 & - & $<4.7$ & 2.7 \\
\hline 79 & 2003 Jun. 06-09 & $52796.88-52797.07$ & 14 & - & - & - \\
\hline 82 & 2003 Jun. $15-18$ & $52805.94-52806.15$ & 14 & 2.1 & $3.2 \pm 0.3$ & 9.5 \\
\hline $87 \dagger$ & 2003 Jun. 30-Jul 03 & $52820.92-52821.08$ & 12 & 6.5 & $5.1 \pm 0.4$ & 11.2 \\
\hline 92 & 2003 Jul. 15-18 & $52835.84-52836.00$ & 12 & - & - & - \\
\hline 142 & 2003 Dec. $12-14$ & $52985.44-52985.60$ & 12 & - & - & - \\
\hline 145 & 2003 Dec. $21-23$ & $52994.41-52994.62$ & 12 & - & $<7.6$ & 2.9 \\
\hline 153 & 2004 Jan. $14-16$ & $53019.25-53019.45$ & 14 & - & - & - \\
\hline 162 & 2004 Feb. 10-12 & $53045.49-53045.69$ & 14 & - & - & - \\
\hline 177 & 2004 Mar. 26-29 & $53090.55-53091.48$ & 16 & - & - & - \\
\hline 181 & 2004 Apr. 07-10 & 53 102.88-53 103.05 & 15 & - & - & - \\
\hline 185 & 2004 Apr. 19-22 & $53114.56-53114.70$ & 13 & - & - & - \\
\hline 189 & 2004 Apr. 30-33 & $53126.47-53126.63$ & 15 & - & $<4.4$ & 4.8 \\
\hline 193 & 2004 May 12-15 & $53138.39-53138.42$ & 4 & - & - & - \\
\hline 202 & 2004 Jun. 08-11 & $53165.48-53165.67$ & 15 & - & - & - \\
\hline 210 & 2004 Jul. 02-05 & $53189.33-53189.51$ & 15 & - & - & - \\
\hline 229 & 2004 Aug. 29-Sep. 01 & $53246.96-53247.13$ & 15 & - & - & - \\
\hline 233 & 2004 Sep. 09-12 & $53258.01-53258.80$ & 8 & - & - & - \\
\hline 234 & 2004 Sep. 12-15 & $53260.99-53261.92$ & 17 & - & - & - \\
\hline
\end{tabular}

\subsection{Radio observations}

We observed 4U 2206+54 with the NRAO 4 Very Large Array (VLA) at $8.4 \mathrm{GHz}(3.6 \mathrm{~cm}$ wavelength) on two different epochs: May 12, 2003 from 7:05 to 8:00 and from 11:40 to 12:52 UT (average MJD 52771.4, during INTEGRAL

${ }^{4}$ The National Radio Astronomy Observatory is a facility of the National Science Foundation operated under cooperative agreement by Associated Universities, Inc. revolution 70) with the VLA in its D configuration, and May 20, 2003 from 15:27 to 17:20 UT (MJD 52779.7, during INTEGRAL revolution 73) with the VLA during the reconfiguration from $\mathrm{D}$ to $\mathrm{A}$. The observations were conducted as follows: 10-min scans on $4 \mathrm{U} 2206+54$, preceded and followed by 2 -min scans on the VLA phase calibrator $2250+558$. The primary flux density calibrator used was $1331+305$ (3C 286). The data were reduced using standard procedures within the NRAO AIPS software package. 

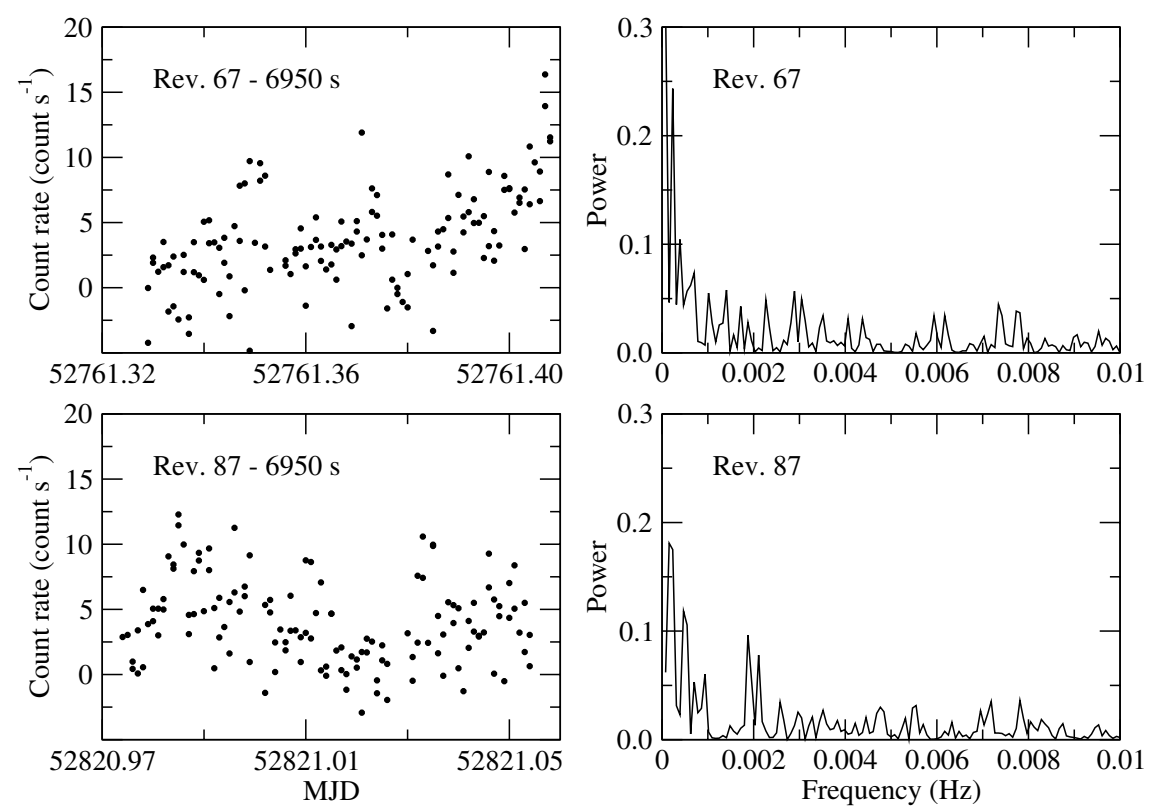

Fig. 1. ISGRI lightcurves of $4 \mathrm{U} 2206+54$ in the energy range $20-40 \mathrm{keV}$ binned at $50 \mathrm{~s}$ (left) and associated power spectra (right) for a time span equivalent to 3 INTEGRAL science windows (around $6950 \mathrm{~s}$ ) during revolutions 67 (top panels) and 87 (bottom panels).

\section{Results}

\subsection{High energies}

\subsubsection{Timing}

Analysis of the X-ray lightcurves clearly shows that the source is variable on all timescales. However, except from the $9.6 \mathrm{~d}$ modulation observed in RXTE/ASM data (see Corbet \& Peele 2001; Ribó et al. 2005) and believed to be the orbital period, no other periodic variability has been detected so far. Unfortunately, INTEGRAL coverage of the source is not enough to test the presence of the orbital periodicity. Therefore, orbital period analysis is outside the scope of this paper.

Pulse period analysis gave negative results for both our ISGRI and JEM-X datasets. This was expected, as previous searches on similar timescales had also failed (see NR01, Corbet \& Peele 2001; Torrejón et al. 2004; Masetti et al. 2004).

ISGRI data from consecutive pointings were joined together when possible and rebinned to $50 \mathrm{~s}$ to search for possibly longer periods. Nothing was found up to periods of $\sim 1 \mathrm{~h}$. The 20-40 keV lightcurve and power spectrum for a time-span of 6950 s during revolutions 67 and 87 can be seen in Fig. 1. The difference between the two lightcurves and between the corresponding power spectra is apparent. A quasi-periodic feature at $\sim 0.002 \mathrm{~Hz}(\sim 500 \mathrm{~s})$ can be seen in data from revolution 87 , but it is not present at other epochs. The timing behaviour of the source seems to be different in every pointing.

Little attention has been paid so far to intermediate periods (on the order of hours), perhaps because intermediate periods would be difficult to detect in the RXTE/ASM data, especially when points are filtered and rebinned as one-day averages to keep statistical significance. We therefore searched the $R X T E / P C A$ lightcurves described in Sect. 2.1 for intermediate period pulsations to test the possible presence of a slowly rotating NS. Unfortunately, the gaps due to the satellite low-Earth orbit are rather large in comparison to the periods searched, which certainly hampers the search somewhat. We used epoch folding and Lomb-Scargle periodogram techniques with negative results, and in Fig. 2 show the power spectrum analysis of the whole time span. No significant period was detected, particularly in the interval [0.95-5.5] $\times 10^{-4} \mathrm{~Hz}$, which corresponds to periods from $3 \mathrm{~h}$ to $30 \mathrm{~min}$, approximately.

Searching inside individual observations, we found some periodicities, although with low significance. Inside the RXTE/PCA 60071-01-03 observation we find a possible period of $6900 \mathrm{~s}$ while in observation 60071-01-04 we find $8620 \mathrm{~s}$. Folding the entire lightcurve on either of these periods results in no pulsed signal. We conclude therefore that analysis of the entire lightcurve does not deliver any significant period in the range explored. This result leaves only the possibility of a period in the range from some hours to $1 \mathrm{~d}$ to be explored. In order to do this, a suitably long observation with a high Earth orbit satellite, like INTEGRAL, would be required.

We show in Fig. 3 the long-term lightcurves of $4 \mathrm{U} 2206+54$ in different energy ranges, from the $2-12 \mathrm{keV}$ of the RXTE/ASM data up to $80 \mathrm{keV}$ for the ISGRI data, spanning $120 \mathrm{~d}$. As can be seen in the $20-40 \mathrm{keV}$ lightcurve, an increase in brightness occurred during revolution 67 (MJD 52 761.36). The source brightness increased threefold over a timespan on the order of half an hour (see also the top left panel in Fig. 1).

\subsubsection{Spectral analysis}

From the whole timespan during which ISGRI collected data from 4U 2206+54, the source was inside both the Fully Coded Field Of View (FCFOV) of ISGRI and the JEM-X FOV only during one pointing in revolution 67 and 3 pointings in revolution 87 . Therefore, the available spectrum from revolution 67 

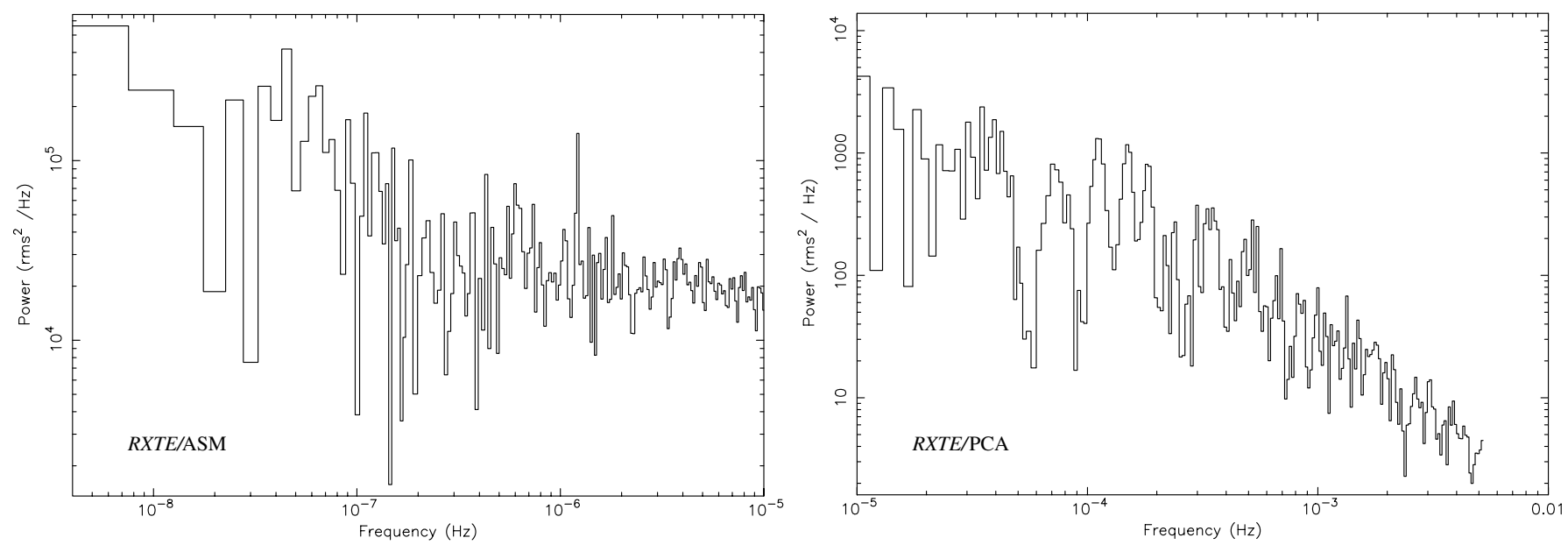

Fig. 2. Left: power spectrum of the RXTE/ASM lighcurve, binned at $25 \mathrm{ks}$ intervals. Note the feature at a frequency of $\simeq 1.2 \times 10^{-6} \mathrm{~Hz}$, which corresponds to the orbital period (see Corbet \& Peele 2001). No other period is found at the limit of the lightcurve resolution. Right: power spectrum of the $100 \mathrm{ks}$ RXTE/PCA lightcurve, binned at $96 \mathrm{~s}$ intervals. No significant period is detected in the frequency interval [0.95-5.5] $\times$ $10^{-4} \mathrm{~Hz}$, which corresponds approximately to the time interval from $3 \mathrm{~h}$ to $30 \mathrm{~min}$. The slope of the power spectrum is $\sim v^{-0.8}$, where $v$ is the frequency.

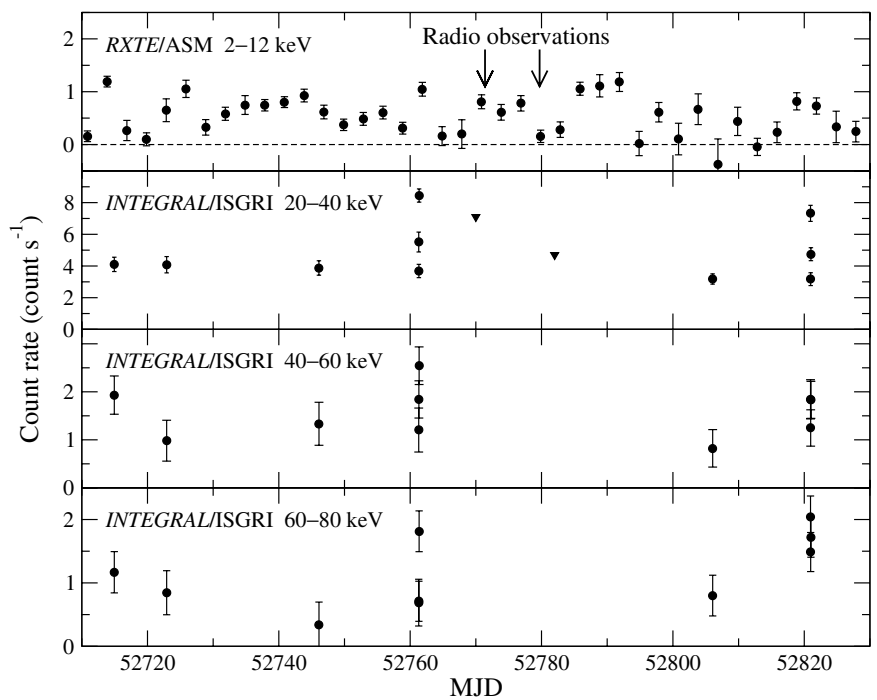

Fig. 3. Lightcurves of $4 \mathrm{U} 2206+54$ in different energy bands (quoted inside the different panels). The top panel is for the 3-day average of the RXTE/ASM data, where the arrows indicate the epochs of radio observations. The three bottom panels are for the INTEGRAL/ISGRI data in intervals of $\sim 2 \mathrm{ks}$. Error bars represent the mean of the 1-day average data errors for $R X T E / A S M$ data and the formal errors for INTEGRAL/ISGRI data. The two triangles in the $20-40 \mathrm{keV}$ lightcurve indicate $3 \sigma$ upper limits.

and the mean spectrum from revolution 87 were used for the spectral analysis. Systematic errors of $10 \%$ for ISGRI and $5 \%$ for JEM-X were added to our data sets in order to perform a more realistic spectral analysis ${ }^{5}$. The software package used was XSPEC 11.2 (Arnaud 1996).

In order to compare with previously published data, the comptonisation model of Sunyaev \& Titarchuk (1980), as improved by Titarchuk (1994) to include relativistic effects, implemented in XSPEC as compTT, and a powerlaw model,

\footnotetext{
${ }^{5}$ Private communication from the teams of the instruments.
}

modified to include photon absorption and a high energy cutoff, were chosen to fit the data. For the comptonisation model, the emitting region temperatures derived were $10 \pm 3$ and $13 \pm$ $8 \mathrm{keV}$ for the data of revolutions 67 and 87, respectively. The fits were acceptable, with corresponding $\chi_{\text {Red }}^{2}$ of 1.3 for 173 degrees of freedom (DOF) in the first observation and $\chi_{\text {Red }}^{2}$ of 1.2 for 176 d.o.f. in the second observation. The powerlaw parameters of both observations are listed in Table 2.

Both models yield a $4-150 \mathrm{keV}$ flux of $\sim 16 \times$ $10^{-10} \mathrm{erg} \mathrm{s}^{-1} \mathrm{~cm}^{-2}$ for revolution 67 and $\sim 8 \times 10^{-10} \mathrm{erg} \mathrm{s}^{-1} \mathrm{~cm}^{-2}$ for revolution 87 . Assuming a distance to the source of $3 \mathrm{kpc}$ (NR01), its luminosity amounts to $\sim 1.7 \times 10^{36} \mathrm{erg} \mathrm{s}^{-1}$ and $\sim 0.9 \times 10^{36} \mathrm{erg} \mathrm{s}^{-1}$, respectively. Around $\sim 50 \%$ of the total luminosity lies in the $4-12 \mathrm{keV}$ energy band, that is, $\sim 8.5 \times 10^{35} \mathrm{erg} \mathrm{s}^{-1}$ for revolution 67 and $\sim 4.5 \times 10^{35} \mathrm{erg} \mathrm{s}^{-1}$ for revolution 87 . We notice that during these observations the source appears brighter than in any previous observation. The RXTE/ASM lightcurve confirms that the flux was high in the $2-12 \mathrm{keV}$ band as well.

We show the INTEGRAL spectra of 4 U 2206+54 in Fig. 4 for revolutions 67 and 87 . Both spectra suggest the presence of an absorption feature around $\sim 30 \mathrm{keV}$, as already noticed by Torrejón et al. (2004) and Masetti et al. (2004) in RXTE and BeppoSAX data, see Table 2. An absorption feature through the cyclabs model (in XSPEC notation) was added to the powerlaw model. In the revolution 67 spectrum, the absorption feature was fitted at an energy of $32 \pm 5 \mathrm{keV}$ for a fixed line width of $3 \mathrm{keV}$. The same feature is apparent in the spectrum from revolution 87 , where it was fitted at $32 \pm 3 \mathrm{keV}^{6}$. Except for the normalization factors, the fitted parameters to the datasets

${ }^{6}$ An F-test was applied to the spectral fits of revolution 87, which has a better signal to noise ratio than that of revolution 67. Improving of the $\chi^{2}$ by the inclusion of a cyclabs component has a $12 \%$ probability of ocurring by chance. One should take into account the limitations of this test when applied to lines (Protassov et al. 2002). 
Table 2. Comparison of the most recently published powerlaw+high-energy cutoff parameters, adding photon absorption at lower energies, for $4 \mathrm{U} 2206+54$. We also refer the reader to Tables 2-4 of Saraswat \& Apparao (1992) for earlier EXOSAT data. All fluxes shown are unabsorbed in the quoted energy ranges. The errors are at a 68\% confidence level for the entry of Negueruela \& Reig (2001) and at 90\% confidence level for the rest of the data except for the entry of Corbet \& Peele (2001), where confidence levels were not reported.

\begin{tabular}{|c|c|c|c|c|c|c|c|c|}
\hline Ref. (Mission, year) & $\Gamma$ & $\begin{array}{c}E_{\text {cut }} \\
(\mathrm{keV})\end{array}$ & $\begin{array}{c}E_{\text {fold }} \\
(\mathrm{keV})\end{array}$ & $\begin{array}{c}N_{\mathrm{H}} \times 10^{22} \\
\left(\text { atom } \mathrm{cm}^{-2} \text { ) }\right.\end{array}$ & $\begin{array}{c}\chi_{\text {Red }}^{2} \\
\text { (d.o.f.) }\end{array}$ & $\begin{array}{r}\text { Flux } \times 10^{-10} \\
\left(\mathrm{erg} \mathrm{s}^{-1} \mathrm{~cm}^{-2}\right)\end{array}$ & $\begin{array}{l}\text { Energy range } \\
(\mathrm{keV})\end{array}$ & $\begin{array}{c}E_{\text {cycl }} \\
(\mathrm{keV})\end{array}$ \\
\hline Negueruela \& Reig 2001 (RXTE, 1997) & $1.7 \pm 0.3$ & $7.4 \pm 0.2$ & $17.5 \pm 0.8$ & $4.7 \pm 0.2$ & $0.9(56)$ & 4.8 & $2.5-30$ & not reported \\
\hline Corbet \& Peele 2001 (RXTE, 1997-1) & $1.71 \pm 0.03$ & $7.3 \pm 0.1$ & $17.3 \pm 0.6$ & $4.6 \pm 0.2$ & $0.82^{a}$ & 3.1 & $2-10$ & not reported \\
\hline$(R X T E, 1997-2)$ & $1.12 \pm 0.12$ & $5.3 \pm 0.2$ & $10.5 \pm 1.2$ & $2.7 \pm 0.7$ & $0.75^{a}$ & 1.1 & $2-10$ & not reported \\
\hline Torrejón et al. $2004(R X T E, 1997)$ & $1.6 \pm 0.1$ & $7.6 \pm 0.4$ & $16.3 \pm 1.2$ & $4.5 \pm 0.4$ & $0.71(52)$ & 2.7 & $2-10$ & not reported \\
\hline$(R X T E, 2001)$ & $1.6 \pm 0.1$ & $4.3 \pm 0.3$ & $20 \pm 2$ & $4.6 \pm 0.1$ & $1.27(49)$ & 1.3 & $2-10$ & $29^{b}$ \\
\hline (BeppoSAX, 1998) & $1.0 \pm 0.2$ & $7.8 \pm 0.5$ & $11 \pm 3$ & $1.1 \pm 0.3$ & $1.32(113)$ & 0.4 & $2-10$ & $35 \pm 5^{b}$ \\
\hline Masetti et al. 2004 (BeppoSAX, 1998) & $0.95_{-0.14}^{+0.11}$ & $4.3_{-0.5}^{+0.6}$ & $10.6_{-2.0}^{+2.7}$ & $0.88_{-0.19}^{+0.21}$ & $1.1(219)$ & 0.4 & $2-10$ & $35 \pm 5^{c}$ \\
\hline This paper (INTEGRAL, 2003, Rev. 67) & $1.8 \pm 0.7$ & $13 \pm 5$ & $22 \pm 6$ & 1.0 (fixed) & $1.2(154)$ & 15.9 & $4-150$ & $32 \pm 5$ \\
\hline This paper (INTEGRAL, 2003, Rev. 87) & $1.7_{-0.4}^{+0.3}$ & $11 \pm 5$ & $29_{-7}^{+8}$ & 1.0 (fixed) & $1.0(153)$ & 8.3 & $4-150$ & $32 \pm 3$ \\
\hline
\end{tabular}

${ }^{a}$ No information about the DOF is reported in the reference. ${ }^{b}$ No significance reported. ${ }^{c}$ At $2 \sigma$ confidence level.
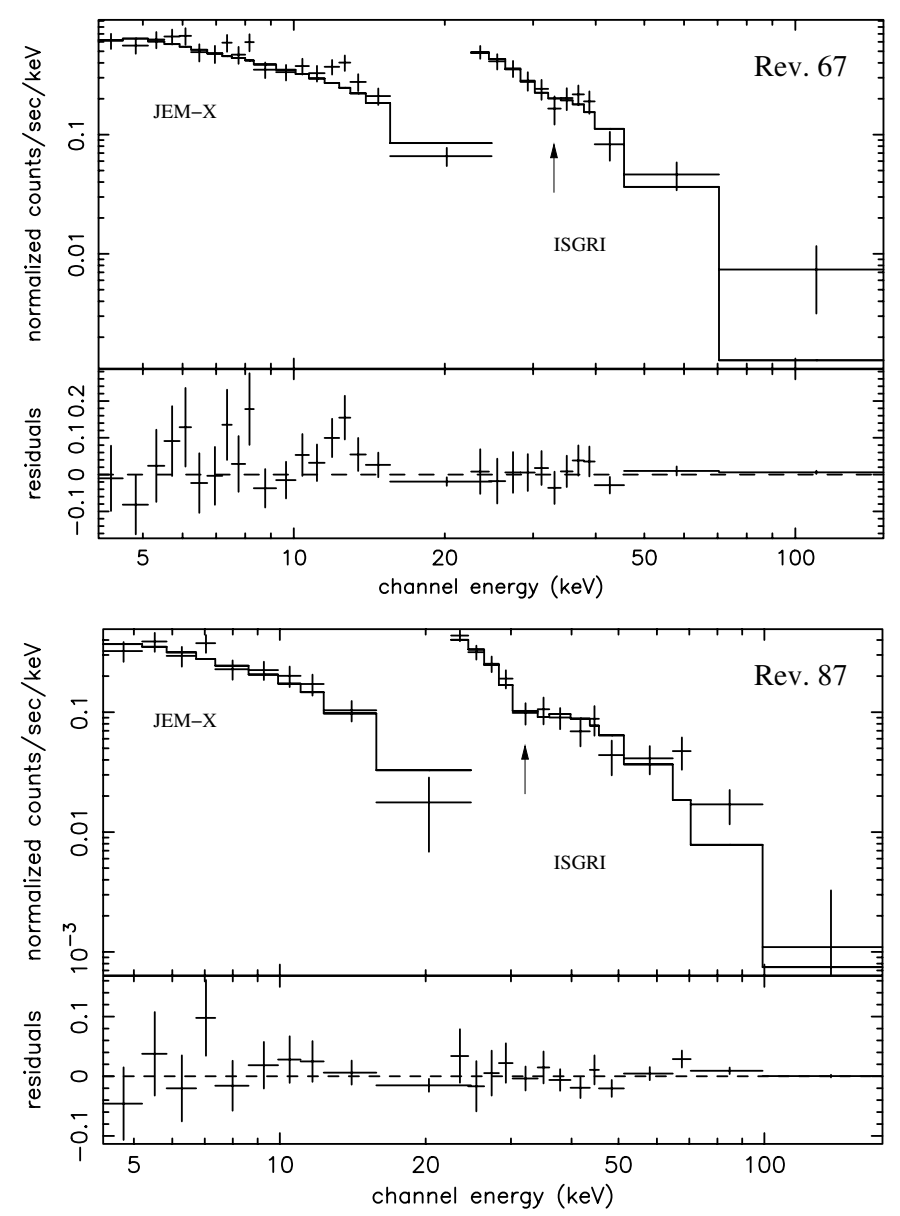

Fig. 4. Spectra of 4U 2206+54 from JEM-X and ISGRI detectors. Top: the spectrum shown is for $2.2 \mathrm{ks}$ of exposure time during revolution 67 (MJD 52 761.36). Bottom: the spectrum shown is for $6.6 \mathrm{ks}$ of exposure time during revolution 87 (MJD 52 821.17). In both cases the spectral model shown in Table 2 is represented by the solid line, and the residuals to the model are displayed in the lower panels. The presence of an absorption feature around $32 \mathrm{keV}$, indicated by arrows, is suggested by the two datasets. of both revolutions are compatible between them within the errors (see Table 2).

There are well-known calibration problems in the ISGRI Response Matrix Function (RMF) that may cast some doubt on the reality of the absorption feature reported here. In order to investigate if this feature is an instrumental effect, we normalised the $20-60 \mathrm{keV}$ spectra of $4 \mathrm{U} 2206+54$ and Crab to their respective continua modeled by a powerlaw, and then divided the normalised $4 \mathrm{U} 2206+54$ spectrum over that of the Crab. We have chosen a Crab observation as close in time as possible to our data and with similar off-axis angles, to ensure that the RMF and off-axis effects are as similar as possible. In Fig. 5 we show the observed spectra (top panel), their ratio to the powerlaw model (middle panel), and the ratio between the former ratios (bottom panel). The absorption feature around $32 \mathrm{keV}$ is still seen. The quality of the data does not allow us to state that the detection is statistically significant, but the likely presence of a feature at this position had already been reported in the analysis of two other independent datasets obtained by two different satellites (see Table 2). As it has been seen by three different instruments at different times, the existence of this absorption feature is strongly suggested. Such features in X-ray spectra are generally attributed to Cyclotron Resonance Scattering Features (CRSFs) (see, e.g., Coburn et al. 2002, and references therein).

Motivated by the possible presence of this CRSF, we summed up images from those INTEGRAL revolutions with significant ISGRI detections. The effective exposure time of this mosaic amounts to $\sim 27 \mathrm{ks}$, and we show its extracted spectrum in Fig. 6. We note that spectral shape changes with luminosity have been reported in Saraswat \& Apparao (1992) and NR01. Therefore, by summing up data taken on different epochs we might be losing spectral shape information. However, our goal is not to study the shape of the continuum, but to achieve an improved signal to noise ratio at the CSRF position, which is suggested by both the revolution 67 and 


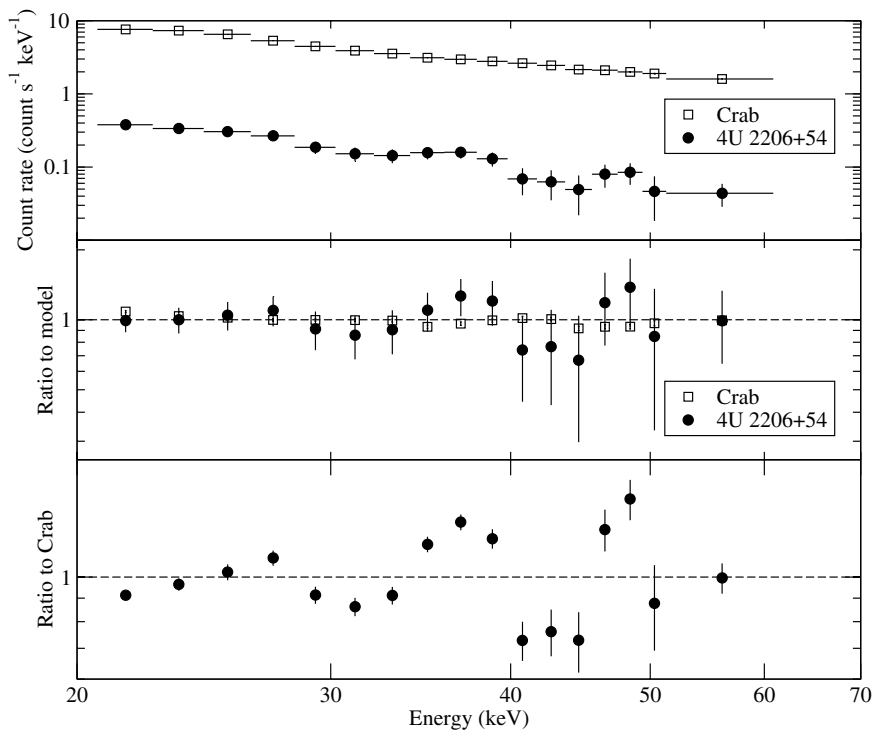

Fig. 5. Observed ISGRI spectra of the Crab and 4U 2206+54 during revolution 67 (top panel), their ratio to the powerlaw model (middle panel), and the ratio of $4 \mathrm{U} 2206+54$ to that of the Crab for the former ratios. The variations above $40 \mathrm{keV}$ are not smooth, but random and show large errors, indicating that they most likely reflect extraction problems due to the fact that we are approaching the sensitivity limit of ISGRI $\left(\sim 0.04\right.$ count $\mathrm{s}^{-1} \mathrm{keV}^{-1}$ for $2 \mathrm{ks}$ exposures and $3 \sigma$ level detection at $45 \mathrm{keV})$.

revolution 87 spectra to be at $\sim 32 \mathrm{keV}$. The best fit to the continuum of the new spectrum was a comptonisation model of soft photons by matter undergoing relativistic bulk-motion, i.e. bmc in XSPEC notation (Shrader \& Titarchuk 1999), which provided a $\chi_{\text {Red }}^{2}$ of 1.7 for 6 degrees of freedom (see top panel in Fig. 6). We added a CSRF absorption by using the XSPEC cyclabs, at $32 \pm 2 \mathrm{keV}$, to the bmc model, and obtained a slightly improved fit with $\chi_{\text {Red }}^{2}$ of 1.3 for 5 degrees of freedom. Finally, we also fitted the data by adding a Lorentzian profile in absorption to the bmc model. In this case the $\chi_{\text {Red }}^{2}$ lowered to 1.1 for 5 degrees of freedom (see bottom panel in Fig. 6). The center of the line was located at $31.5 \pm 0.5 \mathrm{keV}$ and its $F W H M$ was found to be $0.015 \pm 0.005 \mathrm{keV}$. Normalization of the line, $1.5_{-0.8}^{+0.7} \times 10^{-3}$ count s${ }^{-1} \mathrm{keV}^{-1}$ at a $68 \%$ confidence level $(1 \sigma)$, yields a significance of the line of $\sim 2 \sigma^{7}$. We note that the significance of this detection might somehow be influenced by changes in the continuum during the different observations. On the other hand, although the significance depends as well on the model chosen to fit the continuum, any model that properly fits the obtained continuum will reveal the presence of $\sim 2 \sigma$ absorption around $32 \mathrm{keV}$, as can be seen from the data in Fig. 6. Thus we can conclude that the presence of a CRSF is strongly suggested by the data. This result, when combined with previous claims based on BeppoSAX and RXTE

\footnotetext{
7 The significance of the CRSF detection could not be derived from the individual analysis of revolutions 67 and 87, due to poor improvement in the statistics obtained when adding the CRSF component in both cases. We can only state the significance of the detection after nice improvement in the signal-to-noise ratio achieved by the mosaic.
}
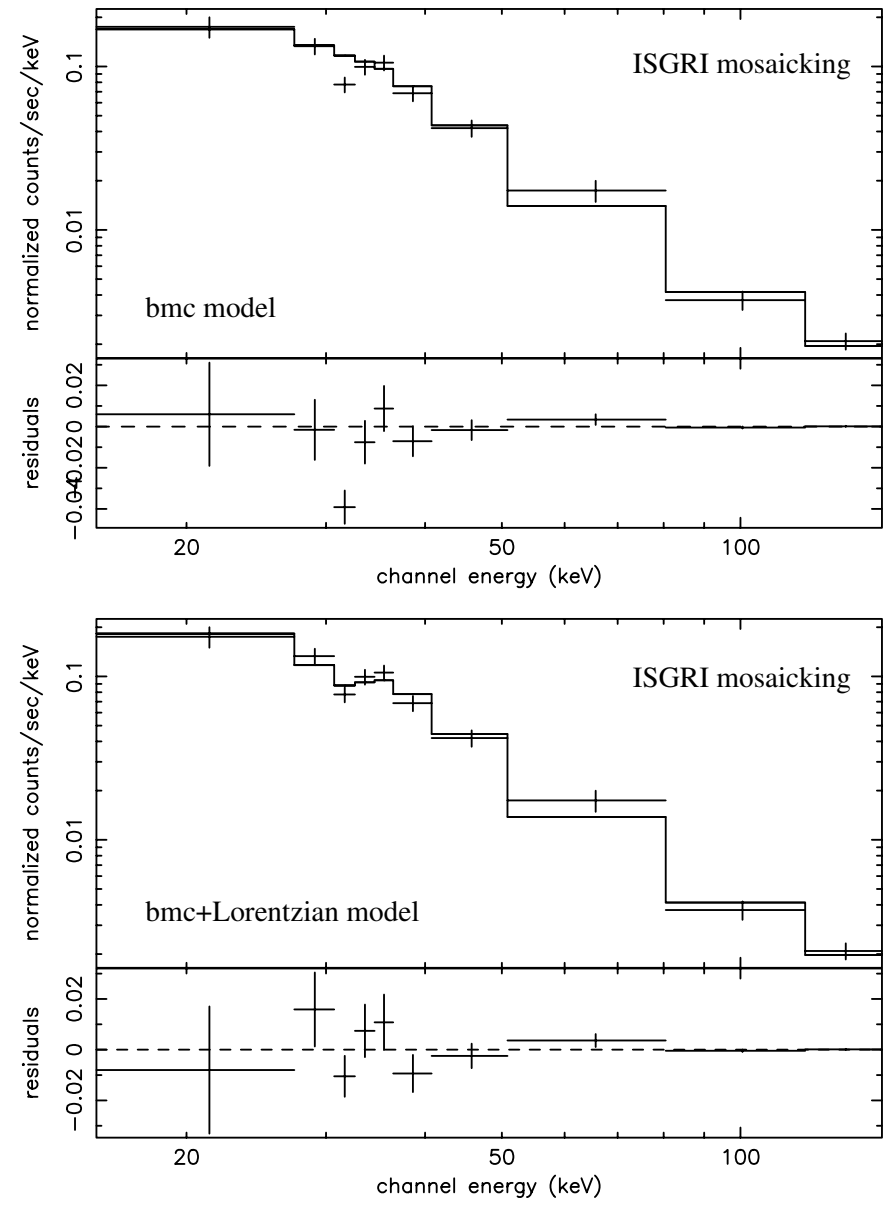

Fig. 6. Top: spectrum of $4 \mathrm{U} 2206+54$ extracted from the mosaicking of all images with significant detections from ISGRI. The solid line represents the bmc model spectrum (in XSPEC notation) fitted to the data. An absorption feature around $32 \mathrm{keV}$ is seen in the residuals. Bottom: the same extracted spectrum, but here the solid line represents the bmc model plus a Lorentzian feature in absorption fitted to the data, yielding a line position of $31.5 \pm 0.5 \mathrm{keV}$.

data (Torrejón et al. 2004; Masetti et al. 2004) gives evidence for the presence of this CSRF in $4 U 2206+54$.

\subsection{Radio}

No radio emission at $8.4 \mathrm{GHz}$ was detected, with a $3 \sigma$ upper limit of $0.042 \mathrm{mJy}$ on May 12, 2003 (MJD 52 771.4) and a $3 \sigma$ upper limit of $0.066 \mathrm{mJy}$ on May 20, 2003 (MJD 52 779.7). We concatenated all the data and obtained a final $3 \sigma$ upper limit of $0.039 \mathrm{mJy}$. The resulting image is shown in Fig. 7.

\section{Discussion}

\subsection{Conflicts in the neutron star scenario}

The aperiodic variability of the X-ray emission from $4 \mathrm{U} 2206+54$ favours the idea that the X-ray source is powered by wind-fed accretion onto a compact object. The X-ray luminosity of the source combined with its X-ray spectral shape most likely excludes the possibility of a white dwarf (see, e.g., de Martino et al. (2004) for accreting white dwarf 


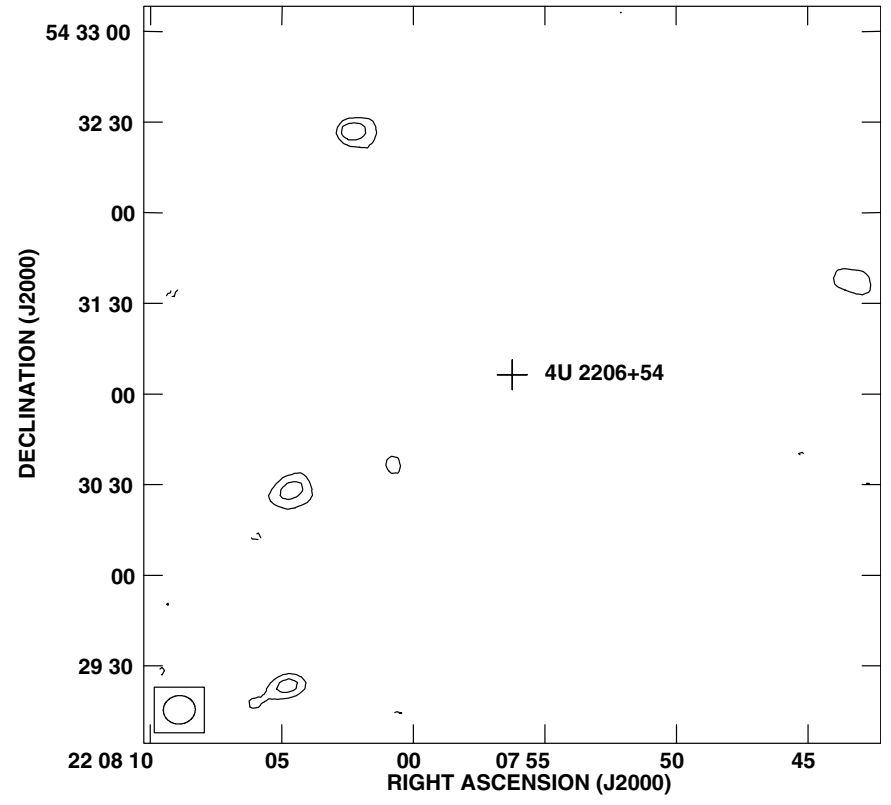

Fig. 7. Image around $4 U 2206+54$, marked with a cross, obtained with the VLA at $8.4 \mathrm{GHz}$ after concatenating data from May 12 and 20, 2003. The image size is $4^{\prime} \times 4^{\prime}$. Contours are $-3,3$, and 5 times the rms noise level of $0.013 \mathrm{mJy}_{\text {beam }}{ }^{-1}$. The ellipse in the bottom left corner represents the Full Width Half Maximum of the obtained synthesised beam of $10.6^{\prime \prime} \times 9.4^{\prime \prime}$ in $\mathrm{PA}=-83.4^{\circ}$.

$\mathrm{X}$-ray spectra). The compact object must be, therefore, a neutron star or black hole.

There are two main difficulties for accepting 4U 2206+54 as a typical wind-accreting neutron star. The first one is the lack of pulsations, as most other wind-fed systems are X-ray pulsars. This could result from a geometrical effect: if the angle between the spin axis and the magnetic axis of the neutron star is close to zero or if the whole system has a very low inclination angle, all the high-energy radiation seen could be coming from a permanently observed single pole of the neutron star. The system inclination is unlikely to be very small, as the projected $v \sin i$ for the optical companion is not particularly small (NR01), unless there is a very strong misalignment between the rotation axis of the optical star and the orbit. However, if the angles between the spin and magnetic axes of neutron stars are drawn from a random distribution, there is a non-negligible chance that for some systems they will be aligned. Similar scenarios have been proposed to explain the absence of pulsations from 4U 1700-37 (White et al. 1983) and also from the lowmass X-ray binary 4U 1700+24 (Masetti et al. 2002), though in the latter case there is no conclusive evidence that this system is sufficiently young to show pulsations.

The second, stronger argument is the expected X-ray luminosity. We can consider a canonical neutron star with $10 \mathrm{~km}$ radius and $1.4 M_{\odot}$ accreting from the fast wind of a low-luminosity O9 III-V star in a $9.6 \mathrm{~d}$ orbit. Following the Bondi-Hoyle approximation, the accretion luminosity obtained, which is an upper limit to the $\mathrm{X}$-ray luminosity, is $\sim 1 \times$ $10^{34} \mathrm{erg} \mathrm{s}^{-1}$ (see Reig et al. 2003 for details about the method). In contrast, the observed X-ray luminosity of 4U 2206+54 is in the range $\sim 10^{35}-10^{36} \mathrm{erg} \mathrm{s}^{-1}$, therefore comparable to those of HMXBs with OB supergiant donors (see Negueruela 2004, and references therein), which are believed to have massloss rates more than one order of magnitude higher than an O9 III-V star (Leitherer 1988; Howarth \& Prinja 1989). In addition, we note that our estimate for the semimajor axis of $4 \mathrm{U} 2206+54$, of $55-60 R_{\odot}$, is comparable to the highest values of semimajor axes in supergiant systems (see Kaper et al. 2004). Therefore, a close orbit cannot be invoked to solve the problem of the high X-ray luminosity.

\subsection{Excluding the black hole scenario}

If a black hole was present in the system, the photon index and luminosities of our INTEGRAL observations would indicate that the source is in a low/hard state (see McClintock \& Remillard 2004, and references therein). Our radio observations took place on May 12 and 20, 2003 or during INTEGRAL revolutions 70 and 73, i.e., right between INTEGRAL observations at revolutions 67 (May 01-04, 2003) and 87 (Jun. 30-Jul. 03, 2003).

Gallo et al. (2003) found an empirical correlation between the soft X-ray flux (in the range $2-11 \mathrm{keV}$ ) and the centimetre radio emission (with observed flat spectrum in the range 4.9-15 GHz) for black hole binary systems in the low/hard state of the form: $S_{\text {radio }}=(223 \pm 156) \times\left(S_{\mathrm{X}}\right)^{+0.7}$, where $S_{\text {radio }}$ is the radio flux density scaled to $1 \mathrm{kpc}, S_{\mathrm{X}}$ the X-ray flux in Crab units scaled to $1 \mathrm{kpc}$, and the uncertainty in the multiplying factor is the non-linear $1 \sigma$ error of their fit. Therefore, by using a measured X-ray flux we can compute the expected radio emission of a source in case it is a black hole.

We obtained the flux from $4 \mathrm{U} 2206+54$ in the 2-11 keV band from our JEM-X data, being 7.2 and $4.0 \times$ $10^{-10} \mathrm{erg} \mathrm{s}^{-1} \mathrm{~cm}^{-2}$ for the May and June 2003 observations, respectively. This flux was translated to Crab units by measuring the flux from the Crab in the $2-11 \mathrm{keV}$ band, using JEM-X data from an INTEGRAL observation close in time to our $4 \mathrm{U} 2206+54$ pointings. The Crab flux was found to be $1.8 \times 10^{-8} \mathrm{erg} \mathrm{s}^{-1} \mathrm{~cm}^{-2}$, leading to fluxes of 40 and $22 \mathrm{mCrab}$ for $4 \mathrm{U} 2206+54$ during revolutions 67 and 87, respectively. From this, and using $N_{\mathrm{H}}=1.0 \times 10^{22}$ atom $\mathrm{cm}^{-2}$ (average of the values obtained by Torrejón et al. 2004 and Masetti et al. 2004 from BeppoSAX data), we computed the unabsorbed corrected flux following Eq. (1) of Gallo et al. (2003), and then the resulting flux in Crab units scaled to $1 \mathrm{kpc}$ distance (assuming a distance of $3 \mathrm{kpc}$ to $4 \mathrm{U} 2206+54$ ). The relation discussed above then predicts a radio flux density, already scaled back again to $3 \mathrm{kpc}$, of $12.6 \pm 8.8 \mathrm{mJy}$ at the time of revolution 67 and of $8.3 \pm 5.8 \mathrm{mJy}$ for revolution 87 , where the errors come directly from the $1 \sigma$ uncertainties given in Gallo et al. (2003) for the parameters of their fit. Thus, for revolution 67 the expected radio emission would be in the range 3.8-21.4 $\mathrm{mJy}$ and for revolution 87 in the range $2.5-14.2 \mathrm{mJy}$.

We note that the lower expected radio flux density of $2.5 \mathrm{mJy}$ is already more than 60 times greater than the $0.039 \mathrm{mJy} 3 \sigma$ upper limit found with our VLA observations. Obviously, it can be argued that our observations were not simultaneous. During revolution 70, which was in 
coincidence with the first radio observations, the flux found from ISGRI data is not significant enough and, unfortunately, the source was outside the FOV of JEM-X, which could have provided a suitable X-ray flux for this analysis. Nevertheless, we point out that the RXTE/ASM count rate during our first radio observation is very similar to that measured during revolution 87 , so it is reasonable to compare the obtained $2.5 \mathrm{mJy}$ limit with our measured $0.042 \mathrm{mJy} 3 \sigma$ upper limit on that day, giving again a difference of a factor $\sim 60$. It could also be argued that the source could have experienced a transition to the high/soft state, which would naturally prevent the detection of radio emission. However, in such a case the RXTE/ASM count rates should have increased considerably, while during both radio observations the count rates were similar or lower than during revolutions 67 and 87, when the photon indexes were typical of low/hard states. In summary, if the correlation between $\mathrm{X}$-ray emission and radio emission indeed reflects a general property of black hole systems, we conclude that there is not a black hole in 4U 2206+54.

Moreover, Fender \& Hendry (2000) show that all persistent Galactic black holes have detectable radio emission. As $4 \mathrm{U} 2206+54$ is a persistent source and does not show any detectable radio emission, it cannot be hosting a black hole. Systems containing magnetised neutron stars $\left(B \gtrsim 10^{11} \mathrm{G}\right)$, on the other hand, do not show detectable radio emission.

\subsection{The cyclotron feature}

The indication of a cyclotron feature centred at $32 \mathrm{keV}$ strongly suggests that there is a magnetic neutron star in $4 \mathrm{U} 2206+54$, in good agreement with the lack of radio emission. INTEGRAL is the third mission reporting the likely detection of this absorption feature (see Table 2) and, even if none of the detections can be considered statistically significant, the fact that it appears in three independent datasets cannot be ignored.

If the line is indeed a CRSF we can compute the value of the magnetic field in the scattering region by means of the equation $\left[B / 10^{12} \mathrm{G}\right]=\left[E_{\text {cycl }} / 11.6 \mathrm{keV}\right](1+z)$, with $z$ the gravitational redshift at which we see the region. Considering that the line is produced at the surface of a canonical neutron star of $1.4 M_{\odot}$ with a radius of $10 \mathrm{~km}$, the gravitational redshift amounts to $z=0.3$ (see, e.g., Kreykenbohm et al. 2004), and from the position of the line centre at $32 \mathrm{keV}$ we obtain a magnetic field of $3.6 \times 10^{12} \mathrm{G}$. This value, in agreement with those found by Torrejón et al. (2004) and Masetti et al. (2004), is typical of magnetic neutron stars, and well within the range of 1.3-4.8 $\times$ $10^{12} \mathrm{G}$ obtained by Coburn et al. (2002) for a sample of ten $\mathrm{X}$-ray pulsars displaying CRSFs (see their Table 7).

One is led to the conclusion that $4 \mathrm{U} 2206+54$ is the first system known in which an accreting magnetic neutron star does not appear as an X-ray pulsar. The possibility of very slow pulsations cannot be directly discarded. The wind-accreting X-ray source $2 \mathrm{~S} 0114+650$ with a B1 supergiant donor (Reig et al. 1996) shows pulsations with a period of $\sim 2.8 \mathrm{~h}$ (Finley et al. 1994). The orbital period of the system is $\sim 12 \mathrm{~d}$, similar to that of $4 \mathrm{U} 2206+54$. Our timing analysis rules out the possibility of significant pulsations from $4 \mathrm{U} 2206+54$ in the range $0.5-3.0 \mathrm{~h}$.
Modulation at a period of several hours is still possible, as existing datasets do not constrain this period range strongly. However, it seems more logical to conclude that geometrical effects are responsible for the lack of pulsations.

\section{Conclusions}

We present the first INTEGRAL GPS results on 4U $2206+54$ together with contemporaneous VLA observations of the source. A broad high-energy spectrum (4-150 keV), joining JEM-X and ISGRI data, has been extracted and fitted with spectral models similar to those used in the analysis of previously published data obtained with other satellites. The evidence for the presence of a cyclotron line is strengthened, as INTEGRAL becomes the third high-energy mission to observe a possible feature at $32 \pm 3 \mathrm{keV}$. If the feature is indeed a cyclotron line, it indicates a magnetic field strength of $3.6 \times 10^{12} \mathrm{G}$, which is typical of magnetised neutron stars.

Our VLA radio observations failed to detect the source at a very low level, indicating that any possible radio emission is at least 60 times weaker than what would be expected from a black hole system in the low/hard state. This lack of radio detection is again compatible with the presence of a magnetised neutron star. 4U 2206+54 appears to be the first known system containing an accreting neutron star that does not show up as an $\mathrm{X}$-ray pulsar, which is most likely due to a simple geometrical effect.

Longer high-energy exposures, such as an INTEGRAL long pointing, are needed to improve the $S / N$ ratio to a level that will allow confirmation of the presence of the cyclotron feature and study of its eventual profile and energy changes with time and luminosity. Such observation would also allow the search for very long (several hours) or very weak pulsations.

Acknowledgements. We are grateful to the VLA Scheduling Committee, which allowed us to conduct the observations as an ad hoc proposal. We thank Silvia Martínez Núñez for all the useful discussions about JEM-X data and Elena Gallo for useful clarification of the X-ray/radio correlation. We acknowledge useful comments and clarifications from Nicolas Produit and INTEGRAL Science Data Center members. We acknowledge the anonymous referee for detailed and useful comments that helped to improve the paper. This research is supported by the Spanish Ministerio de Educación y Ciencia (former Ministerio de Ciencia y Tecnología) through grants AYA2001-3092, ESP-2002-04124-C03-02, ESP-200204124-C03-03, and AYA2004-07171-C02-01, partially funded by the European Regional Development Fund (ERDF/FEDER). P.B. acknowledges support by the Spanish Ministerio de Educación y Ciencia through grant ESP-2002-04124-C03-02. M.R. acknowledges support by a Marie Curie Fellowship of the European Community programme Improving Human Potential under contract number HPMF-CT-200202053. I.N. is a researcher of the programme Ramón y Cajal, funded by the Spanish Ministerio de Educación y Ciencia and the University of Alicante, with partial support from the Generalitat Valenciana and the European Regional Development Fund (ERDF/FEDER). This research has made use of the NASA Astrophysics Data System Abstract Service and of the SIMBAD database, operated at the CDS, Strasbourg, France. 


\section{References}

Arnaud, K. A. 1996, in Astronomical Data Analysis Software and Systems V, ASP Conf. Ser., 101, 17

Bildsten, L., Chakrabarty, D., Chiu, J., et al. 1997, ApJS, 113, 367

Clark, J. S., Reig, P., Goodwin, S. P., et al. 2001, A\&A, 376, 476

Clark, J. S., Goodwin, S. P., Crowther, P. A., et al. 2002, A\&A, 392, 909

Coburn, W., Heindl, W. A., Rothschild, R. E., et al. 2002, ApJ, 580, 394

Condon, J. J., Cotton, W. D., Greisen, E. W., et al. 1998, AJ, 115, 1693

Corbet, R. H. D., \& Peele, A. G. 2001, ApJ, 562, 936

Diehl, R., Baby, N., Beckmann, V., et al. 2003, A\&A, 411, L117

de Martino, D., Matt, G., Belloni, T., Haberl, F., \& Mukai, K. 2004, A\&A, 419, 1009

Fender, R. P., \& Hendry, M. A. 2000, MNRAS, 317, 1

Finley, J. P., Taylor, M., \& Belloni, T. 1994, ApJ, 429, 356

Gallo, E., Fender, R. P., \& Pooley, G. G. 2003, MNRAS, 344, 60

Goldwurm, A., David, P., Foschini, L., et al. 2003, A\&A, 411, L223

Howarth, I. D., \& Prinja, R. K. 1989, ApJS, 69, 527

Jahoda, K., Swank, J. H., Giles, A. B., et al. 1996, in EUV, X-ray, and Gamma-Ray Instrumentation for Astronomy VII, ed. O. H. Siegmund, \& M. A. Gummin, SPIE, 2808, 59

Kaper, L., van der Meer, A., \& Tijani, A. H. 2004, in Proc. of IAU Coll., 191, Revista Mexicana de Astronomía y Astrofísica (Serie de Conferencias), 21, 128

Kreykenbohm, I., Wilms, J., Coburn, W., et al. 2004, A\&A, 427, 975

Leitherer, C. 1988, ApJ, 326, 356

Maraschi, L., \& Treves, A. 1981, MNRAS, 194, 1P

Martocchia, A., Motch, C., \& Negueruela, I. 2005, A\&A, 430, 245

Masetti, N., Dal Fiume, D., Cusumano, G., et al. 2002, A\&A, 382, 104

Masetti, N., Dal Fiume, D., Amati, L, et al. 2004, A\&A, 423, 311

Massi, M., Ribó, M., Paredes, J. M., et al. 2004, A\&A, 414, L1
McClintock, J. E., \& Remillard, R. A. 2004, in Compact Stellar X-Ray Sources, ed. W. H. G. Lewin, \& M. van der Klis (Cambridge University Press), in press [arXiv: astro-ph/0306213]

McSwain, M. V., Gies, D. R., Huang, W., et al. 2004, ApJ, 600, 927

Negueruela, I., \& Reig, P. 2001, A\&A, 371, 1056 (NR01)

Negueruela, I. 2004, in proceedings of The Many Scales of the Universe-JENAM 2004 Astrophysics Reviews, Kluwer Academic Publishers, ed. J. C. del Toro, \& Iniesta et al. [arXiv: astro-ph/0411759]

Paredes, J. M., Martí, J., Ribó, M., \& Massi, M. 2000, Science, 288, 2340

Paredes, J. M., Ribó, M., Ros, E., Martí, J., \& Massi, M. 2002, A\&A 393, L99

Protassov, R., van Dyk, D. A., Connors, A., Kashyap, V. L., \& Siemiginowska, A. 2002, ApJ, 571, 545

Reig, P., Chakrabarty, D., Coe, M. J., et al. 1996, A\&A, 311, 879

Reig, P., Ribó, M., Paredes, J. M., \& Martí, J. 2003, A\&A, 405, 285

Ribó, M., Reig, P., Martí, J., \& Paredes, J. M. 1999, A\&A, 347, 518

Ribó, M., Negueruela, I., Torrejón, J. M., Blay, P., \& Reig, P. 2005, A\&A, submitted

Saraswat, P., \& Apparao, K. M. V. 1992, ApJ, 401, 678

Shrader, C. R., \& Titarchuk, L. 1999, ApJ, 521, L121

Skinner, G., \& Connell, P. 2003, A\&A, 411, L123

Sunyaev, R. A., \& Titarchuk, L. G. 1980, A\&A, 86, 121

Titarchuk, L. 1994, ApJ, 434, 570

Torrejón, J. M., Kreykenbohm, I., Orr, A., Titarchuk, L., \& Negueruela, I. 2004, A\&A, 423, 301

Westergaard, N. J., Kretschmar, P., Oxborrow, C. A., et al. 2003, A\&A, 411, L257

White, N. E., Swank, J. H., \& Holt, S. S. 1983, ApJ, 270, 711

Winkler, C., Courvoisier, T. J.-L., Di Cocco, G., et al. 2003, A\&A, 411, L1 